\title{
Scaling test of the P4-improved staggered fermion action.
}

\section{Michael Cheng* for the RBC-Bielefeld Collaboration}

Columbia University

E-mail: michaelc@phys.columbia.edu

\begin{abstract}
We present a scaling study of the QCD spectrum using a smeared P4 staggered fermion formulation, in which three, five, and seven-link staples are added to reduce the effects of flavor symmetry breaking. These studies are performed on quenched lattices generated using the oneloop improved Symanzik gauge action, with $\beta=\frac{10}{g^{2}}=7.40,7.75,8.00$. The corresponding lattice spacings are $\mathrm{a}=.31 \mathrm{fm}, .21 \mathrm{fm}$, and $.14 \mathrm{fm}$. Particularly, we study the $O\left(a^{2}\right)$ flavor symmetry violations in the pion spectrum as well as the scaling dependence of $m_{\rho}$, measured in physical units, on lattice spacing. These results are compared against previous results for the Asqtad action.
\end{abstract}

XXIIIrd International Symposium on Lattice Field Theory

25-30 July 2005

Trinity College, Dublin, Ireland

${ }^{*}$ Speaker. 


\section{Introduction}

Lattice QCD is an important method for computing interesting quantities in the Standard Model as well as in finite temperature QCD. However, strictly speaking, the different lattice formulations of QCD reproduce the continuum theory only in the limit that the lattice spacing goes to zero $(a \rightarrow 0)$. Thus, it is important to choose a lattice action where the finite lattice spacing errors are as small as possible. One of the more popular improved fermion actions is the Asqtad action [1]. It has been shown from quenched measurements of various quantities that the Asqtad action has much smaller scaling errors than many other unimproved actions[2].

Another interesting improved action is the $\mathrm{P} 4$ action[3]. In these proceedings we study the scaling properties of the $\mathrm{P} 4$ action, particularly as compared to Asqtad. To accomplish this, we make quenched measurements of the Goldstone and non-Goldstone pion masses. The mass splitting between these two particles is one manifestation of $O\left(a^{2}\right)$ effects in the staggered formulation[4]. We also look at how $m_{\rho}$ scales with lattice spacing, and the effect of tadpole improvement[5] on all of these quantities.

\section{P4 action}

Like the Asqtad action, the P4 action is a variant of the Kogut-Susskind formulation of lattice fermions. To improve the naive staggered action, we allow the inclusion of higher order derivatives. There are two possible three-link terms allowed by the hypercubic symmetry of the staggered formulation. These are the familiar Naik term[6], and the so-called "knight's move" term:

$$
\begin{aligned}
S_{F}= & m \sum_{x} \bar{\chi}(x) \chi(x)+\sum_{x} \bar{\chi}(x) \sum_{\mu} \eta_{\mu}(x) \\
& \left\{c_{1,0}\left[U_{\mu}(x) \chi(x+\mu)-U_{\mu}^{\dagger}(x-\mu) \chi(x-\mu)\right]+\right. \\
& +c_{3,0}\left[U_{\mu}^{(3,0)}(x) \chi(x+3 \mu)-U_{\mu}^{(3,0) \dagger}(x-3 \mu) \chi(x-3 \mu)\right]+ \\
& +c_{1,2} \sum_{v \neq \mu}\left[U_{\mu, v}^{(1,2)}(x) \chi(x+\mu+2 v)-U_{\mu, v}^{(1,2) \dagger}(x-\mu-2 v) \chi(x-\mu-2 v)+\right. \\
& \left.\left.+U_{\mu, v}^{(1,-2)}(x) \chi(x+\mu-2 v)-U_{\mu, v}^{(1,-2) \dagger}(x-\mu+2 v) \chi(x-\mu-2 v)\right]\right\}
\end{aligned}
$$

where $\chi(x)$ is the one-component fermion field, $\eta_{\mu}(x)$ is the staggered phase, and the various parallel transporters are given by

$$
\begin{aligned}
U_{\mu}^{(3,0)}(x) & =U_{\mu}(x) U_{\mu}(x+\mu) U_{\mu}(x+2 \mu) \\
U_{\mu, v}^{(1,2)}(x) & =\frac{1}{2}\left[U_{\mu}(x) U_{v}(x+\mu) U_{v}(x+\mu+v)+U_{v}(x) U_{v}(x+v) U_{\mu}(x+2 v)\right] \\
U_{\mu, v}^{(1,-2)}(x) & =\frac{1}{2}\left[U_{\mu}(x) U_{v}^{\dagger}(x+\mu-v) U_{v}^{\dagger}(x+\mu-2 v)+U_{v}^{\dagger}(x-v) U_{v}^{\dagger}(x-2 v) U_{\mu}(x-2 v)\right]
\end{aligned}
$$

If we examine the naive quark propagator $\left(c_{1,0}=1, c_{3,0}=0, c_{1,2}=0\right)$, we see that rotational symmetry is violated at $O\left(p^{4}\right)$. However, with an improved staggered action, we can systematically eliminate these violations if the coefficients satisfy the constraint $c_{1,0}+27 c_{3,0}-18 c_{1,2}=0$. Coupled with an overall continuum normalization, this produces a one-parameter family of actions that 


\begin{tabular}{cccccccc} 
Action & $c_{1}$ & $c_{\text {Naik }}$ & $c_{\text {knight }}$ & $c_{3}$ & $c_{5}$ & $c_{7}$ & $c_{\text {Lepage }}$ \\
\hline Asqtad & $\frac{1}{8}+\frac{3}{8}+\frac{1}{8}$ & $-\frac{1}{24 u_{0}^{2}}$ & 0 & $\frac{1}{16 u_{0}^{2}}$ & $\frac{1}{64 u_{0}^{4}}$ & $\frac{1}{384 u_{0}^{6}}$ & $-\frac{1}{16 u_{0}^{4}}$ \\
P4fat7 & $\frac{1}{8}-\frac{1}{4}$ & 0 & $\frac{1}{24}$ & $\frac{1}{16}$ & $\frac{1}{64}$ & $\frac{1}{384}$ & 0 \\
P4fat7tad & $\frac{1}{8}-\frac{1}{4}$ & 0 & $\frac{1}{24 u_{0}^{2}}$ & $\frac{1}{16 u_{0}^{2}}$ & $\frac{1}{64 u_{0}^{4}}$ & $\frac{1}{384 u_{0}^{6}}$ & 0
\end{tabular}

Table 1: Fermion action coefficients. $c_{\text {Lepage }}$ is introduced in the Asqtad action to remove the $O\left(a^{2}\right)$ errors introduced by fat link smearing [7]. We choose not to include it in either variant of the P4 action.

\begin{tabular}{|cccccc|}
\hline$\beta=10 / g^{2}$ & $a[\mathrm{fm}]$. & $r_{1} / a$ & plaquette & plaq $^{\frac{1}{4}}$ & input $u_{0}$ \\
\hline 7.40 & .31 & $1.44(1)$ & $.554799(6)$ & $.863045(2)$ & .8629 \\
7.75 & .21 & $2.08(5)$ & $.599179(7)$ & $.879811(2)$ & .8800 \\
8.00 & .14 & $2.65(1)$ & $.621287(4)$ & $.887816(1)$ & .8879 \\
\hline
\end{tabular}

Table 2: Lattice spacing $a$, string tension scale $r_{1}$, and plaquette for the quenched evolutions

remove the leading-order rotational symmetry violation in the quark propagator. The improvement coefficients satisfy $c_{1,2}-c_{3,0}=\frac{1}{24}$.

If we set $c_{1,2}=0, c_{3,0}=-\frac{1}{24}$, we recover the Naik action. The choice $c_{1,2}=\frac{1}{24}, c_{3,0}=0$ yields the $\mathrm{P} 4$ action. Not only does the Naik action improve the rotational symmetry, but it also removes all $O\left(a^{2}\right)$ errors in the staggered propagator [6]. However, if one examines the free-field dispersion relation, one sees that it is the $\mathrm{P} 4$ action that more effectively removes higher-order corrections, thus providing a much better approximation to the free-field, continuum dispersion relation[3].

\section{Smearing and Tadpole Improvement}

The Asqtad action incorporates not only the Naik term, but also makes use of fat-link smearing[8] with tadpole-improved coefficients to reduce the effects of flavor-changing interactions. In order to take full advantage of any possible flavor-symmetry improvement, we incorporate fat-link smearing into our versions of the P4 action. Specifically, we replace the one-link part of the action with the combination

$$
\mathrm{c}_{1} \longrightarrow+\mathrm{c}_{3} \Sigma\left\lceil+\mathrm{c}_{5} \Sigma\lceil\right.
$$

The smearing coefficients are tuned to remove flavor-changing couplings to lowest order in perturbation theory. Furthermore, in order to study the effect of tadpole improvement, we measure the hadron spectrum for two different variants of the P4 action. What we call P4fat 7 utilizes fatlink smearing without tadpole improvement, while P4fat7tad uses tadpole-improved coefficients. Consult Table 1 for a full list of parameters.

\section{Simulation details}

Spectrum measurements were done for the Asqtad, P4fat7, and P4fat7tad actions on three different sets of quenched lattices, chosen to match the lattices used by MILC in their Asqtad scaling study[2]. Lattices were generated using the one-loop tadpole-improved Symanzik gauge 


\begin{tabular}{|c|c|c|c|c|c|c|c|c|c|}
\hline \multirow[b]{2}{*}{ Action } & \multirow[b]{2}{*}{$m_{q} a$} & \multicolumn{4}{|c|}{$\beta=7.40$} & \multicolumn{4}{|c|}{$\beta=7.75$} \\
\hline & & $m_{\pi} a$ & $m_{\pi_{2}} a$ & $m_{\rho} a$ & $m_{\rho_{2}} a$ & $m_{\pi} a$ & $m_{\pi_{2}} a$ & $m_{\rho} a$ & $m_{\rho_{2}} a$ \\
\hline \multirow[t]{4}{*}{ P4fat7 } & .01 & $.4210(3)$ & $.672(9)$ & $1.06(2)$ & $1.09(5)$ & $.3221(5)$ & $.397(3)$ & $.777(12)$ & $.803(19)$ \\
\hline & .02 & $.5842(3)$ & $.797(4)$ & $1.13(1)$ & $1.17(1)$ & $.4500(6)$ & $.505(2)$ & $.819(7)$ & $.835(7)$ \\
\hline & .025 & - & - & - & - & $.5015(6)$ & $.5512(14)$ & $.843(5)$ & $.854(5)$ \\
\hline & .03 & $.7050(3)$ & $.895(3)$ & $1.199(5)$ & $1.231(7)$ & $.5482(7)$ & $.5954(12)$ & $.871(4)$ & $.883(6)$ \\
\hline \multirow[t]{4}{*}{ P4fat7tad } & .02 & $.4229(3)$ & $.634(7)$ & $1.06(2)$ & $1.06(4)$ & $.3467(5)$ & $.409(2)$ & $.766(20)$ & $.801(10)$ \\
\hline & .025 & - & - & - & - & $.3861(5)$ & $.443(2)$ & $.786(12)$ & $.813(11)$ \\
\hline & .03 & $.5143(3)$ & $.707(4)$ & $1.10(1)$ & $1.11(2)$ & .4217(6) & $.477(2)$ & $.810(15)$ & $.820(8)$ \\
\hline & .04 & .5905(4) & $.766(3)$ & $1.132(6)$ & $1.148(7)$ & $.5015(6)$ & $.5512(14)$ & $.843(5)$ & $.854(5)$ \\
\hline \multirow[t]{4}{*}{ Asqtad } & .02 & $.3782(3)$ & $.668(9)$ & $1.14(2)$ & $1.10(2)$ & $.3307(5)$ & $.422(2)$ & $.793(14)$ & $.819(18)$ \\
\hline & .025 & - & - & - & - & $.3686(5)$ & $.4511(18)$ & $.804(10)$ & $.841(20)$ \\
\hline & .03 & $.4606(3)$ & $.719(5)$ & $1.16(1)$ & $1.15(3)$ & .4027(5) & $.481(3)$ & .813(12) & $.838(12)$ \\
\hline & .04 & $.5292(4)$ & $.767(4)$ & $1.18(1)$ & $1.18(2)$ & $.4631(7)$ & $.5310(16)$ & $.841(8)$ & $.860(8)$ \\
\hline \multirow[b]{2}{*}{ Action } & \multirow[b]{2}{*}{$m_{q} a$} & \multicolumn{4}{|c|}{$\beta=8.00$} & & & & \\
\hline & & $m_{\pi} a$ & $m_{\pi_{2}} a$ & $m_{\rho} a$ & $m_{\rho_{2}} a$ & & & & \\
\hline \multirow[t]{3}{*}{ P4fat7 } & .01 & $.2736(5)$ & $.3026(9)$ & $.627(6)$ & $.625(7)$ & & & & \\
\hline & .02 & $.3821(6)$ & $.4025(6)$ & $.666(3)$ & $.670(3)$ & & & & \\
\hline & .03 & $.4662(5)$ & $.4827(5)$ & $.707(2)$ & $.710(2)$ & & & & \\
\hline \multirow[t]{3}{*}{ P4fat7tad } & .01 & $.2170(5)$ & $2519(13)$ & $.605(7)$ & $.605(8)$ & & & & \\
\hline & .02 & $.3025(5)$ & $.3270(8)$ & $.635(5)$ & $.634(5)$ & & & & \\
\hline & .03 & .3679(4) & $.3876(6)$ & $.659(3)$ & $.661(3)$ & & & & \\
\hline \multirow[t]{3}{*}{ Asqtad } & .01 & $.2107(4)$ & $.2558(15)$ & $.619(8)$ & $.607(7)$ & & & & \\
\hline & .02 & $.2941(4)$ & $.3272(8)$ & $.648(5)$ & $.637(5)$ & & & & \\
\hline & .03 & $.3579(6)$ & .3856(8) & $.669(3)$ & $.667(5)$ & & & & \\
\hline
\end{tabular}

Table 3: Hadron masses for $\beta=7.40, \beta=7.75$, and $\beta=8.00$

action with a gauge heat bath evolution. We chose $\beta=\frac{10}{g^{2}}=7.4,7.75$, and 8.00 with volumes of $16^{3} \times 32$ for the first two ensembles and $24^{3} \times 32$ for the latter ensemble.

For the $16^{3} \times 32$ lattices, spectrum measurements were made on 100 configurations, separated by 1000 heat bath sweeps. For the $24^{3} \times 32$ lattices, 100 measurements were made on lattices separated by 500 heat bath sweeps. The hadron spectrum was measured for each fermion action for a set of at least three masses using a $2 \mathbb{Z}$ wall source. All computation was done on QCDOC computers. Table 2 shows the values for the plaquette, the string tension scale $r_{1}$ [2], and the tadpole-improvement coefficient $u_{0}$.

\section{Results}

Table 3 show the result of our spectrum measurements. For each value of $\beta=7.40,7.75$, and 8.00, we measured the mass for the pseudoscalar meson $\left(m_{\pi}\right)$, the scalar meson $\left(m_{\pi_{2}}\right)$, the vector meson $\left(m_{\rho}\right)$, and the pseudovector meson $\left(m_{\rho_{2}}\right)$. As expected, we can see the $O\left(a^{2}\right)$ splitting between the pseudoscalar and scalar pion at all lattice scales. Figure 1 show $m_{\pi}^{2}$ and $m_{\pi_{2}}^{2}$ plotted against $m_{\pi}^{2}$. The straight line through the filled shapes represent $m_{\pi}^{2}$ for each of the three actions. The other three lines through the unfilled shapes are chiral extrapolations for $m_{\pi_{2}}^{2}$. 


\section{Beta $=7.40$}

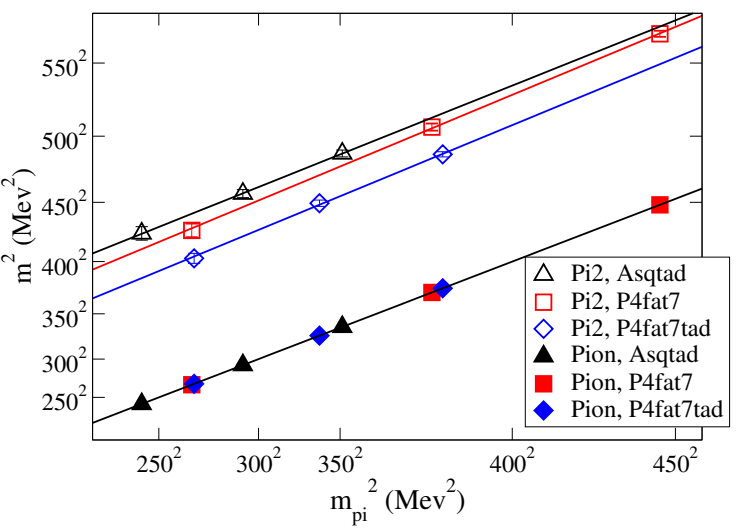

Beta $=8.00$

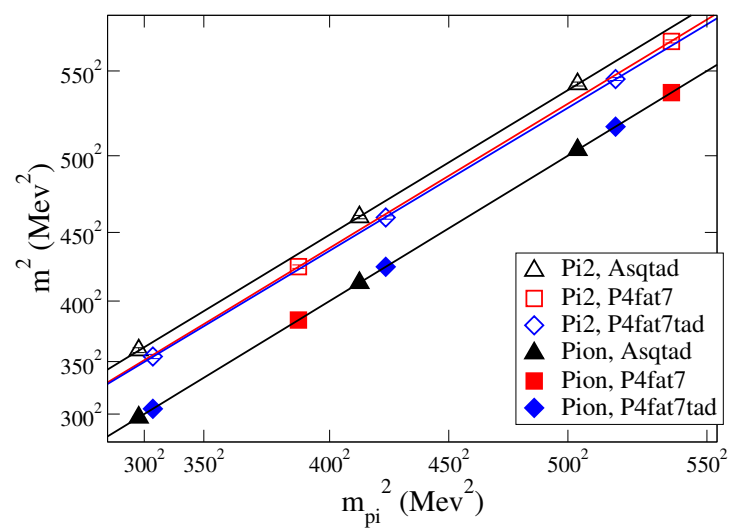

Beta $=7.75$
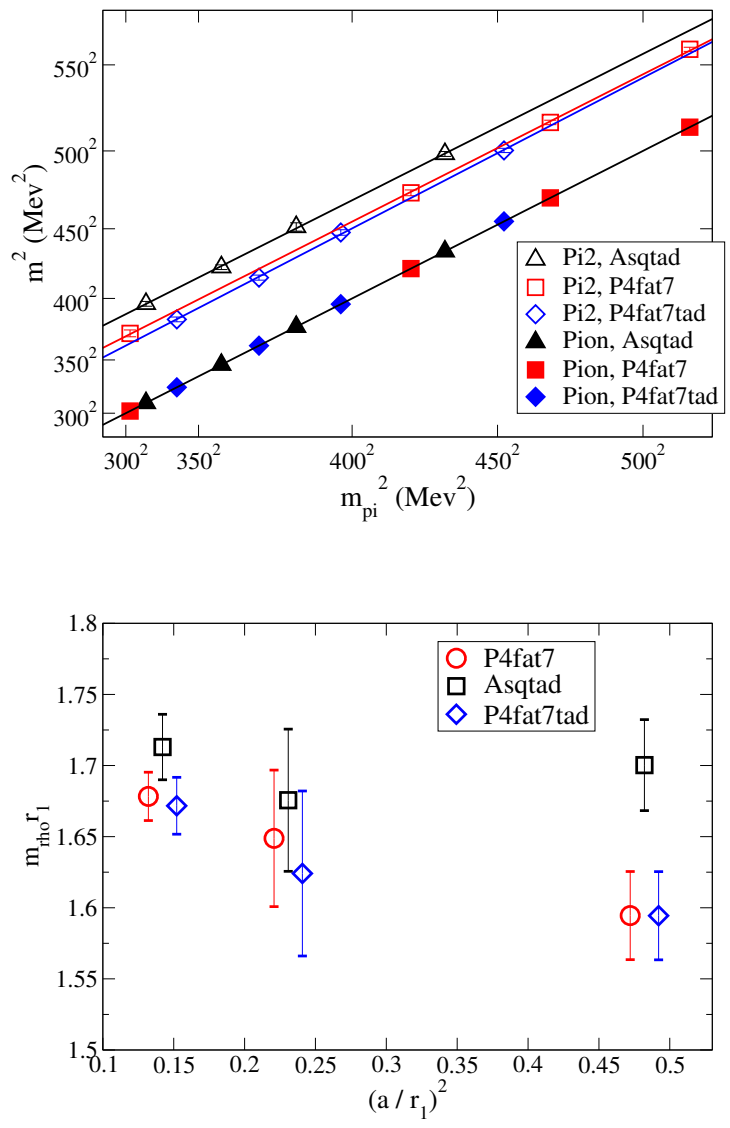

Figure 1: The figures on the top left, top right, and bottom left show $m_{\pi_{2}}^{2}$ vs. $m_{\pi}^{2}$ for $\beta=7.40, \beta=7.75$, and $\beta=8.00$ respectively. In our notation, $\pi_{2}$ denotes the scalar meson channel (non-Goldstone pion) and $\pi$ is the normal pseudoscalar channel. The bottom right figure shows $m_{\rho} r_{1}$ vs. $a^{2} / r_{1}^{2}$ for the various actions (data points offset for clarity).

As expected, the splitting between the two masses is largest on the coarsest lattice, but diminishes as we go to smaller lattice spacing. Also, we can see that the pion splitting is, in all cases, slightly better for both P4fat7(red squares) and P4fat7tad(blue diamonds) as compared to Asqtad(black triangles). Employing tadpole improvement does seem to improve the pion mass splitting at the coarser lattice spacings, but makes no difference for the finest lattice point $(\beta=8.00)$.

The bottom-right plot in Figure 1 also shows the dependence of $m_{\rho}$ on lattice spacing. For this plot, all masses are extrapolated to the common physical point $m_{\pi} r_{1}=.778$. Then, $m_{\rho}$ is plotted against $a^{2}$, with all scales set in units of the string tension scale $r_{1}$. The black squares reproduce MILC results for the Asqtad reasonably well. The red octagons and blue diamonds show the scaling dependence for the P4 actions. P4fat7 and P4fat7tad give very similar answers, showing an approximately $5 \%$ change in $m_{\rho}$ over a factor of 2 in lattice spacing.

\section{Conclusions and Outlook}

In terms of the mass splitting between the non-Goldstone and the Goldstone pion $\left(m_{\pi_{2}}-m_{\pi}\right)$, both variants of the P4 action show less flavor-symmetry breaking than the Asqtad action. Tadpole 
improvement also slightly improves this flavor splitting on the coarsest lattice, but has almost no effect on the two finer lattices that were studied. In terms of the scaling of $m_{\rho}$, Asqtad does better, showing little change from $a \approx .14 \mathrm{fm}$. to $a \approx .31 \mathrm{fm}$. The $\mathrm{P} 4$ action shows an approximately $5 \%$ decrease over this same range.

To further study the scaling behavior of the $\mathrm{P} 4$ action, we plan to compare these results with measurements using the unsmeared $\mathrm{P} 4$ action, to insure that we see the expected improvement that comes from fat-link smearing. Also, it would be useful to look at the entire set of pions whose mass is split by $O\left(a^{2}\right)$ effects. This would provide a more complete picture of the flavor-symmetry violations for each action. The RBC-Bielefeld collaboration is now using the $\mathrm{P} 4$ action in studying thermodynamic quantities. Therefore, it would also be valuable to study the scaling effects for correspondingly coarser, dynamical lattices.

\section{Acknowledgements}

Thanks to Norman Christ, Frithjof Karsch, Peter Petreczky, Konstantin Petrov, and Christian Schmidt for useful and enlightening discussions. Special thanks to Chulwoo Jung for managing the CPS++ software as well as assistance in implementing and testing the simulation code.

We also thank Peter Boyle, Dong Chen, Norman Christ, Mike Clark, Saul Cohen, Calin Cristian, Zhihua Dong, Alan Gara, Andrew Jackson, Balint Joo, Chulwoo Jung, Richard Kenway, Changhoan Kim, Ludmila Levkova, Huey-Wen Lin, Xiaodong Liao, Guofeng Liu, Robert Mawhinney, Shigemi Ohta, Tilo Wettig, and Azusa Yamaguchi for the development of the QCDOC machine and its software. This development and the resulting computer equipment were funded by the U.S. DOE grant DE-FG02-92ER40699, PPARC JIF grant PPA/J/S/1998/00756 and by RIKEN. This work was supported by DOE grant DE-FG02-92ER40699 and we thank RIKEN, BNL and the U.S. DOE for providing the facilities essential for the completion of this work.

\section{References}

[1] MILC Collaboration, K. Orginos, D. Toussaint, and R. L. Sugar, Variants of fattening and flavor symmetry restoration, Phys. Rev. D60 (1999) 054503, [hep-lat/ 9903032 ].

[2] MILC Collaboration, C. W. Bernard et al., Scaling tests of the improved kogut-susskind quark action, Phys. Rev. D61 (2000) 111502, [hep-lat/9912018].

[3] U. M. Heller, F. Karsch, and B. Sturm, Improved staggered fermion actions for qcd thermodynamics, Phys. Rev. D60 (1999) 114502, [hep-lat/9901010].

[4] W.-J. Lee and S. R. Sharpe, Partial flavor symmetry restoration for chiral staggered fermions, Phys. Rev. D60 (1999) 114503, [hep-lat/ 9905023 ].

[5] G. P. Lepage and P. B. Mackenzie, On the viability of lattice perturbation theory, Phys. Rev. D48 (1993) 2250-2264, [hep-lat/9209022].

[6] S. Naik, On-shell improved lattice action for qcd with susskind fermions and asymptotic freedom scale, Nucl. Phys. B316 (1989) 238.

[7] G. P. Lepage, Flavor-symmetry restoration and symanzik improvement for staggered quarks, Phys. Rev. D59 (1999) 074502, [hep-lat/ 9809157 ].

[8] P. Lepage, Perturbative improvement for lattice qcd: An update, Nucl. Phys. Proc. Suppl. 60A (1998) 267-278, [hep-lat/9707026]. 